Revue européenne des sciences sociales

European Journal of Social Sciences

XLI-125 | 2003

Index de la Revue européenne des sciences sociales (1995-2002)

\title{
Bibliographie des écrits de Giovanni Busino de
} 1995 à 2002

Suite des Bibliographies parues dans la « Revue européenne des sciences sociales ", Tome XXVI, 1988, n. 78, pp. 159 à 203 et Tome XXXVI, 1995, n. 100, pp. $48-57$

\section{OpenEdition}

\section{Journals}

Édition électronique

URL : http://journals.openedition.org/ress/558

DOI : $10.4000 /$ ress.558

ISSN : 1663-4446

Éditeur

Librairie Droz

Édition imprimée

Date de publication : 1 janvier 2003

Pagination : 45-53

ISBN : 2-600-00844-6

ISSN : 0048-8046

\section{Référence électronique}

" Bibliographie des écrits de Giovanni Busino de 1995 à 2002 », Revue européenne des sciences sociales [En ligne], XLI-125 | 2003, mis en ligne le 30 novembre 2009, consulté le 01 mai 2019. URL : http:// journals.openedition.org/ress/558; DOI : 10.4000/ress.558 


\title{
BIBLIOGRAPHIE \\ DES ÉCRITS DE GIOVANNI BUSINO \\ DE 1995 A 2002
}

\author{
Suite des Bibliographies parues \\ dans la «Revue européenne des sciences sociales », \\ Tome XXVI, 1988, n. 78, pp. 159 à 203 \\ et Tome XXXVI, 1995, n. 100, pp. 48-57
}

\section{5}

[C. R. :] Dizionario biografico degli Italiani, voll. XLII-XLIII, Roma, Enciclopedia Treccani, 1993, «Bibliothèque d'Humanisme et Renaissance», LVII, 1995, n. I, p. 195.

[C. R.:] Oreste TOMMASINI, La vita e gli scritti di Niccolò Machiavelli nella loro relazione col Machiavellismo, Bologna, Il Mulino, 1994, «Bibliothèque d'Humanisme et Renaissance», LVII, 1995, n. I, p. 215.

[C. R. :] Istvan NEMESKURTY, Nous, les Hongrois. Histoire de Hongrie, Budapest, Akadémiai Kiado, 1994, «Bibliothèque d'Humanisme et Renaissance », LVII, 1995, n. I, pp. 308-309.

Books reviews, in «The European Journal of the History of Economic Thought», vol. 2, n. 1, Spring 1995, pp. 243-245.

Neoclassical Synthesis of Economics and Sociology by M. Morishima, «Revue européenne des sciences sociales », XXXIII, 1995, n. 100, pp. 157-159.

Norberto Bobbio, in: Universalia 1995, Paris, Encyclopaedia Universalis, 1995, pp. 468-470.

Pareto, pensatore d'élite, «Il Giornale», 10 maggio 1995, p. 14.

Questions actuelles de sociologie de la science. Résumés des leçons du cours d'histoire de l'analyse sociologique de l'année académique 1994-1995 (préparés par A. Miéville Garcia, avec la collaboration de I. Honorez et de N. Jacquemet), Lausanne, Institut d'anthropologie et de sociologie, 1995, 204 p., «Cours, séminaires et travaux», n. 15.

L'identità dell' Italia. Le ricerche di Rosario Romeo (1924-1987) tra storiografia e impegno politico, Lausanne, Institut d'anthropologie et de sociologie, 1995, 102 p., «Cours, séminaires et travaux », n. 16.

Alla riscoperta della Nazione, «La voce repubblicana», 20-21 settembre 1995, pp. 4-5.

Contro $i$ paraocchi ideologici, «La voce repubblicana», 4-5 ottobre 1995, pp. 4-5. 
Les sciences sociales et l' histoire, in: Mélanges offerts à Paul Bairoch. Editeurs: B. Etemad, J. Batou, Th. David, Genève, Ed. Passé Présent, 1995, pp. 85-99.

1861, nascita di una Nazione, «La voce repubblicana», 25-26 ottobre 1995, pp. 4-5.

La sociologie aux prises avec les sélections, in: Publications de l'Université de Lausanne: La sélection, Lausanne, Payot, 1995, pp. 41-54.

Rosario Romeo tra storiografia ed impegno politico, «Rivista storica italiana», CVII, agosto 1995, fasc. II, pp. 387-477.

Successi e limiti dell'Italia», «La voce repubblicana», 22-23 novembre 1995, pp. 4-5.

Pratiques sociales et Théories. Les discordes des universitaires, Genève, Droz, 1995, 336 p. ( «Travaux de droit, d'économie, de sciences politiques, de sociologie et d'anthropologie », n. 171).

Egalité, hiérarchies, sélection, inégalités, «Revue européenne des sciences sociales », XXXIII, 1995, n. 102, pp. 102-122.

Bloc-notes d' un sociologue glaneur, «Revue européenne des sciences sociales », XXXIII, 1995, n. 102, pp. 169-199.

Svizzera-Letteratura, in Enciclopedia italiana di scienze, lettere ed arti, V, Appendice, 1979-1992, Roma, Istituto dell'Enciclopedia Italiana, 1995, pp. 377-379.

\section{6}

Anni '70, la libertà in pericolo, «La voce repubblicana », 10-11 gennaio 1996, pp. 4-5.

Raymond Boudon, un sociologue pas comme les autres. Le refus du dogmatisme, «L'Express », 31 janvier 1996, p. 2.

Il ruolo centrale della Nazione, «La voce repubblicana», 31 gennaio-1 febbraio 1996, pp. 4-5.

Jean Starobinski maître de lucidité, «L'Express », 20 février 1996, p. 2.

Vilfredo Pareto. Una teoria critica della scienza della Società. Antologia a cura di Giovanni Busino, Milano, Biblioteca Universale Rizzoli, 1996, 406 pp.

Il primato della storia politica, «La voce repubblicana », 6-7 marzo 1996, pp. 45.

Un programme ambitieux de recherche en sciences sociales. «Demain la Suisse», soit!, «L'Express », 20 avril 1996, p. 2.

Un regard sociologique sur la crise du droit, «Revue européenne des sciences sociales », XXXIV, 1996, n. 104, pp. 215-220.

Diagnostic sur un monde à la dérive, «L'Express », 25 avril 1996, p. 2.

Un ouvrage magistral de Jean-Blaise Grize. Y a-t-il une logique de la communication?, «L'Express», 21 mai 1996, p. 2. 
Les agitateurs d'idées ont-ils disparu? Les intellectuels stérilisés, «L'Express », 3 juillet 1996, p. 2.

Valeurs universelles et relativisme culturel. Introduction, in: Incertaine Planète. Textes des conférences et des entretiens organisés par les trente-cinquièmes Rencontres Internationales de Genève 1995, Boudry-Neuchâtel, La Baconnière, 1996, pp. 235-238.

Mercato contro fascismo (en collaboration avec Pierluigi Ciocca), «Il Sole24 ore », 28 luglio 1996, p. 23.

[Traduction en langue arabe de] Critiques du savoir sociologique, Beyrouth, E.U.P., 1996, 247 p.

Du bon usage de la sociologie. Pavane pour Jean-Claude Passeron. Écrits réunis par Ariane Miéville et Giovanni Busino, «Revue européenne des sciences sociales », XXXIV, 1996, n. 103, 374 pp.

Pour une histoire de la sociologie française. Notes bibliographiques (en collaboration avec Ariane Miéville), «Revue européenne des sciences sociales», XXXIV, 1996, n. 103, pp. 355-371.

Limites du marché et de la science économique. Repenser autrement l'économie, «L'Express», 2 octobre 1996, p. 2.

Science économique. Le vrai Walras, «L'Express», 3 octobre 1996, p. 2.

Mauss : hier et aujourd' hui (édité en collaboration avec Gérald Berthoud). Actes du XII ${ }^{\mathrm{e}}$ Colloque annuel du Groupe d'Etude «Pratiques sociales et Théories », «Revue européenne des sciences sociales», XXXIX, 1996, n. 105, 284 p.

Marcel Mauss, interprète d'un phénomène social total: le Bolchevisme, « Revue européenne des sciences sociales», XXXIV, 1996, n. 105, pp. 75-91.

Politique suisse de la science. En finir avec le flou et les intrigues, «L'Express», 30 octobre 1996, p. 2.

Organisations humanitaires. Le procès, «L'Express», 31 octobre 1996, p. 2.

La sociologie, science emprunteuse ou savoir métaphorique?, in: Groupe de Montheron, Les cigales et les fourmis. Des emprunts entre sciences. Textes réunis et commentés par André Delessert et Jean-Claude Piguet, Lausanne, LEP/Loisirs et Pédagogie, 1996, pp. 124-142.

Elite, in: Dictionnaire de philosophie politique. Publié sous la direction de Philippe Raynaud et Stéphane Rials, Paris, PUF, 1996, pp. 200-203.

Sociologie, in: Dictionnaire de philosophie politique. Publié sous la direction de Philippe Raynaud et Stéphane Rials, Paris, PUF, 1996, pp. 615-622.

Les Rencontres Internationales de Genève, de Jean Starobinski à Georges Nivat, «Revue européenne des sciences sociales», XXXIV, 1996, n. 106, pp. 107-112.

Nazione, Stato, Nazionalismi. Ascesa e vicissitudini, in: Storia d'Europa, vol. V : L'età contemporanea. Secoli XIX-XX. A cura di Paul Bairoch \& Erich J. Hobsbawm, Torino, Einaudi, 1996, pp. 919-960.

Potere politico, potere economico e potere privato, in Storia d'Europa, vol. V: L'età contemporanea. Secoli XIX-XX. A cura di Paul Bairoch \& Erich J. Hobsbawm, Torino, Einaudi, 1996, pp. 1145-1164. 
Préambule, in: Institut d'anthropologie et de sociologie, Rapport d'activité 1996, Lausanne, Unil, 1997, pp. 2-7.

Prises de position publiques du directeur, Ibid., pp. 8-14.

Vilfredo Pareto, il solitario di Céligny, «Banca Popolare di Sondrio (Suisse), Rendiconto annuale 1996 », Bergamo, Gruppo Bancario Banca Popolare di Sondrio, febbraio 1997, pp. 26-28.

I misteri di un fortunato ritrovamento. I copialettere di Pareto, Ibid., p. 31.

Sciences et techniques dans la société. Prologue au colloque. Actes du XIII colloque annuel du Groupe d'Etude «Pratiques sociales et Théories », «Revue européenne des sciences sociales », XXXV, 1997, n. 108, pp. 5-13.

La sociologie : à bout de souffle, à la dérive ou en déroute?, «L'Impartial» et in «L'Express», 16 juin 1997, p. 16, et «Campus», n. 38, juillet-septembre 1997, pp. 22-23.

Une initiation aux énigmes de l'économie, «L'Impartial» et «L'express», 22 juillet 1997, p. 8.

Pour la survie du «Dictionnaire biographique des Italiens», «Bibliothèque d'Humanisme et Renaissance», LIX, n. 3, 1997, pp. 643-644.

L'Université sous la loupe de la politique, des finances, du refuge, «L'Impartial» et «L'Express», 11 septembre 1997, p. 20.

Entre le culturalisme, le sociologisme et le relativisme. Quelques aspects du débat contemporain sur la science, in: Logique, discours et pensée. Mélanges offerts à Jean-Blaise Grize. Textes recueillis par Denis Miéville et Alain Berrendonner avec la collaboration de Christiane Tripet, Berne, Peter Lang, 1997, pp. 325-344.

Signets pour des livres à lire, in «Revue européenne des sciences sociales», XXXV, 1997, n. 109, pp.181-196.

Claudio Magris, ou la pensée d'un Européen sans frontière, «L'Impartial» et «L'Express », 14 octobre 1997, p. 16.

Un témoignage sur Jean-Daniel Candaux journaliste, in: Roger DURAND (éd.), C'est la faute à Voltaire. C'est la faute à Rousseau. Recueil anniversaire pour Jean-Daniel Candaux, Genève, Droz, 1997, pp. 675-683.

Grèce des colonels (suite), «Journal de Genève et Gazette de Lausanne», 22 décembre 1997, p. 2.

Burocracia, in: José Louis OROZCO y Consuelo DAVILA (Coordinadores), Breviario político de la Globalización, Mexico, D.F., Facultad de Ciencias Politicas y Sociales UNAM, 1997, pp. 24-29.

Elite, in: José OROZCO y Consuelo DAVILA (Coordinadores), Breviario político de la Globalización, Mexico, D.F., Facultad de Ciencias Politicas y Sociales UNAM, 1997, pp. 89-100.

La sociologie de la connaissance scientifique. Leçons d'histoire de l'analyse sociologique réunies par Ariane Miéville. Année universitaire 1996-1997, 
Université de Lausanne, Institut d'anthropologie et de sociologie, Collection «Pratiques et théories des sciences et des techniques», I, 1997, 183 p.

[Compte rendu de] Riccardo FAUCCI, L'economista scomodo. Vita e opere di Francesco Ferrara, Palermo, Sellerio Editore, 1995, 316 p., «Rivista storica italiana », CIX, janvier, fasc. I, 1997, pp. 361-364.

[Compte rendu de] Francesco NITTI, Machiavelli nella vita e nelle dottrine, a cura di Stefano Palmieri. Volume secondo, Bologna, Società Editrice Il Mulino, 1996, 439 p. ( «Istituto italiano per gli studi storici, Ristampe anastatiche, $1 »)$, «Bibliothèque d'Humanisme et Renaissance», LIX, n. 2, 1997, pp. 420-421.

[Compte rendu de] JOURNET/MARITAIN, Correspondance. Volume I : 19201929. Edition publiée par la Fondation du Cardinal Journet, Fribourg, Suisse, Editions Universitaires \& Paris, Editions Saint-Paul, 1996, VIII-830 p., «Rivista storica italiana», CIX, maggio 1997, fasc. II, pp. 744-751.

\section{8}

[Recensione a] Laurent TISSOT, Politique, société et enseignement supérieur dans le Canton de Vaud. L'Université de Lausanne, 1890-1916. Préface d'André Lasserre, Lausanne, Payot \& Université, 1996, 400 p., «Rivista storica italiana », CX, fasc. I, gennaio 1998, pp. 333-335.

Et si le jargon scientifique servait parfois à dissimuler le vide des idées?, «Allez savoir!», n. 11, pp. 28-33.

Sociologija: na poslednem izdyxanii, v tupike ili na rasput'e?, in: Gumanitarnye nauki : v poiskax opravadanija, Moscou, RGGU, pp. 34-39.

Remarques interlocutoires en marge du colloque "Mémoire et savoirs à l'ère de l'information», «Revue européenne des sciences sociales», XXXVI, n. 111, pp. 217-222.

Sociologie des sciences et des techniques, Paris, PUF, 128 p. «Que sais-je?», n. 3375.

Si nasce per ricordare, «Notiziario della Banca Popolare di Sondrio», n. 77, agosto, pp. 140-143.

Bobbio Norberto (1909- ), in: Dictionnaire des philosophes. Préface d'André Comte-Sponville, Paris, Encyclopædia Universalis et Albin Michel, pp. 237241.

Dahrendorf Ralf (1929- ), in: Dictionnaire des philosophes. Préface d'André Comte-Sponville, Paris, Encyclopædia Universalis et Albin Michel, pp. 416420.

Avant-propos, in: La valeur de la sociologie. Les travaux de Raymond Boudon. Hommages réunis par $G$. Busino et $B$. Valade, in «Revue européenne de sciences sociales », XXXVI, n. 112, pp. 5-7.

L'antisociologisme de Raymond Boudon, «Revue européenne des sciences sociales », XXXVI, n. 112, pp. 263-272. 
Eux, c'est nous? Introduction, in: $\mathrm{XXXVI}^{\mathrm{e}}$ Rencontres Internationales de Genève 1997, Eux et nous. L'Europe face à ses nouvelles déchirures. Textes des conférences et des discussions, Lausanne, L'Âge d'homme, pp. 71-74.

Dahrendorf Ralf (1929- ), in: Dictionnaire de la sociologie, Paris, Encyclopædia Universalis et Albin Michel, pp. 229-233.

Pareto Vilfredo (1848-1923), in: Dictionnaire de la sociologie, Paris, Encyclopædia Universalis et Albin Michel, pp. 631-635.

Combats pour la sociologie, Université de Lausanne, Institut d'anthropologie et de sociologie, Collection «Cours, séminaires et travaux», n. 19, 180 p.

[Recensione a] JOURNET-MARITAIN, Correspondance. Volume II : 1930-1939. Édition établie par Claude Favez, Jacqueline Favre, Monique Sallès, Dominique et René Mougel. Sous la direction de Pierre Mamie et de Georges Cottier, Fribourg, Editions Universitaires \& Paris, Éditions Saint-Paul, 1997, [VIII+] 1004 p., «Rivista storica italiana», CX, settembre 1998, fasc. III, pp. 1134-1137.

[Interventi in ] Atti del Convegno per la presentazione del Fondo Vilfredo Pareto della Banca Popolare di Sondrio, Milano, Sala Vilfredo Pareto, 9 giugno 1998, Si nasce per ricordare, in «Banca Popolare di Sondrio (Suisse), 1998 Rendiconto annuale », Lugano, Banca Popolare di Sondrio (Suisse) S.A., pp. 44-45 e p. 47.

\section{9}

Elites e elitismo. Traduçao Evaristo Santos, Porto-Portugal, RÉS-Editora, Colecçao-Cultura Geral, 1999, 159 p.

Elite(s) et élitisme, Alger, Casbah Editions, 1999, 127 p. (Collection Approches animée par Mustapha Madi, n. 16).

L'image de l'homme dans les sciences sociales d'aujourd' hui, « Revue européenne des sciences sociales », XXXVII, 1999, n. 113, pp. 21-33.

Les sociologues ont perdu la raison, « Equinoxe. Revue de sciences humaines », n. 21, Printemps 1999, pp. 47-56.

Lectures, interventions, notes polémiques, « Revue européenne des sciences sociales », XXXVII, 1999, n. 113, pp. 269-291.

Lire Pareto aujourd'hui?, in: Sous la direction de Alban BOUVIER, Pareto aujourd' hui, Paris, Presses Universitaires de France, 1999, pp. 23-33.

La microhistoire de Carlo Ginzburg, «Bibliothèque d'Humanisme et Renaissance », LXI, 1999, n. 3, pp. 763-778.

Le retour du «Dictionnaire biographique des Italiens», "Bibliothèque d'Humanisme et Renaissance », LXI, 1999, n. 3, pp. 812-814.

[Compte rendu de] Francesco FORTE, Storia del pensiero dell'economia pubblica. I. Il pensiero antico greco-romano e cristiano; II. Dal Medio Evo al Mercantilismo, Milano, Giuffrè Editore, 1999, XII-562 p. + XII-601 p., « Bibliothèque d'Humanisme et Renaissance », LXI, 1999, n. 3, pp. 814-815. 
Il dibattito sulla scienza nelle ricerche recenti. Dal «Programma forte» all" "Affare Sokal», Rivista storica italiana, CXLI, settembre 1999, fasc. III, pp. 707-756.

[Recensione a] JOURNET-MARITAIN, Correspondance. Volume III : 19401949. Edition établie par Claude Favez, Jacqueline Favre, Monique Sallès et René Mougel. Sous la direction de Pierre Mamie et Georges Cottier, Fribourg, Editions Saint-Augustin \& Paris, Editions Paike et Silence, 1998, 974 p., «Rivista storica italiana », CXLI, settembre 1999, fasc. III, pp. 1103-1107.

L'actualité des travaux de Vilfredo Pareto, « Revue européenne des sciences sociales », XXXVII, 1999, n. 116, pp. 359-380.

In difesa dell' Università, « Notiziario della Banca Popolare di Sondrio », n. 81, dicembre 1999, pp. 116-123.

Umberto Ricci: l'uomo, l'economista (en collaboration avec P. Ciocca), in: U. RICCI, Saggi sul risparmio. Prefazione di Luigi Guiso, Lanciano, Casa Editrice Rocco Carabba, 1999, pp.1-3 (Collana «Economisti abruzzesi », 2).

Notes et documents pour une histoire et une sociologie des prix Nobel scientifiques suisses (en collaboration avec I. Honorez et A. Miéville), Université de Lausanne, Institut d'anthropologie et de sociologie, Collection «Pratiques et théories des sciences et des techniques », V, 1999, 139 p.

Sociedade, in: Enciclopédia Einaudi, volume 38, Sociedade-Civilização, Lisboa, Impresa Nacional-Casa Da Moeda, 1999, pp. 11-64.

Socialização, Ibidem, pp. 65-104.

Papel/Estatuto, Ibidem, pp. 105-124.

Grupo, Ibidem, pp. 125-145.

Comunidade, Ibidem, pp. 146-160.

Elite, Ibidem, pp. 245-270.

Opinião, Ibidem, pp. 271-290.

Conflito, Ibidem, pp. 290-313.

Propaganda, Ibidem, pp. 314-335.

Administração, Ibidem, pp. 336-351.

\section{0}

Notes sur les métaphores fondatrices de la connaissance sociologique, « Revue européenne des sciences sociales », XXXVIII, 2000, n. 117, pp. 69-81.

The signification of Vilfredo Pareto's Sociology, « Revue européenne des sciences sociales », XXXVIII, 2000, n. 117, pp. 217-228.

Causalisme, symétrie et réflexivité. Une lecture des travaux de Carlo Ginzburg, in: L'acteur et ses raisons. Mélanges en l' honneur de Raymond Boudon, Paris, PUF, 2000, pp. 25-42.

Pareto redivivo?, in: C. MALANDRINO, et R.MARCHIONATTI, Economia, Sociologia e Politica nell' opera di Vilfredo Pareto, Firenze, Leo S. Olschki, 2000, pp. 1-24. 
Les sciences et les techniques dans la société, in: M. PORRET, J.-F. FAYET et C. FLUCKIGER (dir.), Guerres et Paix. Mélanges offerts à Jean-Claude Favez, Genève, Georg, 2000, pp. 741-752.

Paroles reçues. Du bon usage des sciences sociales (édité en collaboration avec Gérald Berthoud), Genève, Droz, 2000, 432 p.

Ruggiero Romano: un maître intransigeant et un ami fidèle, in: A. FILIPPI (dir.), Ruggiero Romano, l'Italia, l'Europa, l'America. Studi e contributi in occasione della laurea honoris causa, Camerino, Università degli Studi, 2000, pp. 87-100.

Plaidoyer pour l'Université, " Revue européenne des sciences sociales », XXXVIII, 2000, n. 118, pp. 151-162.

Tra storia e politica. Bibliografia degli scritti di Leo Valiani (1926-1999). A cura di Giovanni Busino, Milano, Fondazione Giangiacomo Feltrinelli, 2000, XLV233 pp.

In memoria di Leo Valiani storico e politico, « Rivista storica italiana », CXII, settembre 2000, fasc. III, pp. 869-926.

La riscoperta di Umberto Ricci economista, « Rivista storica italiana », CXII, settembre 2000, fasc. III, pp. 1059-1072.

Causalismo, simmetria e riflessività : una lettura dei lavori di Carlo Ginzburg, «L'Acropoli », I, n. 3, settembre 2000, pp. 311-327.

Quali significati possiamo attribuire ai processi di «razionalizzazione» della mondializzazione?, «Notiziario della Banca popolare di Sondrio», n. 84, décembre 2000, pp. 4-10.

\section{1}

Quelles significations attribuer aux processus de rationalisation de la mondialisation?, in: D. MERCURE (dir.), Une société-monde? Les dynamiques de la mondialisation. Actes des séances plénières du XVI ${ }^{\mathrm{e}}$ congrès de l'Association internationale des sociologues de langue française, Québec, Les Presses de l’Université Laval, 2001, pp. 167-181.

Ricordo d' un amico e d'un maestro: Leo Valiani (1909-1999), « Notiziario della Banca Popolare di Sondrio », n. 85, avril 2001, pp. 4-7.

Jean-Claude Passeron sociologue et épistémologue, in: J.-L. FABIANI (dir.), Le goût de l' enquête. Pour Jean-Claude Passeron, Paris, L'Harmattan, 2001, pp. 13-35.

Dalla retorica della mondializzazione alle realtà delle mondializzazioni?, «L'Acropoli», II, n. 2, avril 2001, pp.121-134.

Leo Valiani cittadino esemplare, «Nuova Antologia», fasc. 2218, avril-juin 2001, pp. 54-63.

Intorno alle discussioni e ricerche recenti sulla sociologia delle scienze, «Revue européenne des sciences sociales », XXXIX, 2001, n. 120, pp. 145-189.

Plaidoyer pour l'Université, « Esprit», août-septembre 2001, pp. 23-31. 
Un caso interessante: Jean-Claude Passeron, sociologo ed epistemologo, «L'Acropoli », II, n. 5, octobre 2001, pp. 530-542.

Un monde surveillé, « Giornale di storia costituzionale », n. 2/2001, pp. 9-12.

Materiali per la bio-bibliografia d'Umberto Ricci, « Annali della Fondazione Luigi Einaudi », XXXV, 2001, pp. 323-410.

\section{2}

[Luigi Einaudi e la Confederazione Elvetica], in: Luigi Einaudi. Diario svizzero. Presentazione del videofilm di Villi Hermann, Roma, Ente per gli studi monetari, bancari e finanziari Luigi Einaudi, Quaderni di ricerche n. 24, 2002, pp. 21-32.

Fragments d'un livre d'histoire de la sociologie, "Revue européenne des sciences sociales », XL, 2002, n. 122, pp. 67-118.

Un monde surveillé, in: XXXVIIIes Rencontres internationales de Genève 2001, Les grandes mutations. Textes des conférences et des débats, Lausanne, L'Âge d'homme, 2002, pp. 125-132, 150, 153-154.

Postilla al dibattito sulla scienza nelle ricerche recenti, " Rivista storica italiana », CXIX, aprile 2002, fasc. I, pp. 246-256.

Un geografo diverso, «L'Acropoli », III, giugno 2002, n. 3, pp. 320-322.

Una società sotto sorveglianza, « L'Acropoli », III, giugno 2002, n. 3, pp. 328333.

Ruggiero Romano (1923-2002). In memoriam, « Bibliothèque d'Humanisme et Renaissance », LXIV, 2002, n. 2, pp. 377-382.

Introduzione alla lettura dell'opera di Vilfredo Pareto, in: AA. VV., Vilfredo Pareto (1848-1923). L'uomo e lo scienziato. A cura di Gavino Manca. Prefazione di Antonio Fazio. Con una Appendice di 63 lettere inedite del Fondo Vilfredo Pareto della Banca Popolare di Sondrio, Sondrio, Banca Popolare di Sondrio- Milano, Libri Scheiwiller, 2002, pp. 19-68.

Histoire, Philosophie et Sociologie des sciences, "Revue européenne des sciences sociales », XL, 2002, n. 124, pp. 309-317.

Una radiografia dello storico Renzo De Felice. In margine d'un libro recente, « Rivista storica italiana », CXIV, dicembre 2002, fasc. III, pp. 951-999.

Ruggiero Romano (1923-2002). In memoriam, «Rivista storica italiana », CXIV, dicembre 2002, fasc. III, pp. 1069-1076. 